\title{
Lymphadénopathie associée à la maladie des griffes du chat
}

\author{
Guifeng Chen MD, Zhongjie Li MD, Guodong Ding PhD
}

Citation : CMAJ 2021 June 21;193:E962. doi : 10.1503/cmaj.202663-f

Voir la version anglaise de l'article ici : www.cmaj.ca/lookup/doi/10.1503/cmaj.202663

$\mathbf{U}$

$\mathrm{n}$ garçon de 10 ans a été dirigé vers le département de pédiatrie pour douleur et œdème croissants au coude droit depuis une dizaine de jours et une faible fièvre intermittente. II n'avait aucun autre symptôme constitutionnel, tel que perte de poids, diaphorèse nocturne ou malaise, et ses symptômes ne s'étaient pas améliorés à la suite d'une antibiothérapie orale par cefprozil $(15 \mathrm{mg} / \mathrm{kg} / \mathrm{j}, 2$ fois par jour pendant 6 jours). L'enfant a déclaré avoir été mordu à l'index droit par un chat errant 4 semaines auparavant et présentait une lésion résiduelle de $0,5 \mathrm{~cm}$. Ses ganglions lymphatiques supra- et épitrochléaires étaient hypertrophiés et sensibles, et il avait des ganglions plus petits, mais sensibles à l'aisselle ipsilatérale. Nous n'avons pas observé d'hépatosplénomégalie ni d'autres lymphadénopathies. Des échographies de la partie supérieure du bras et de l'aisselle droite ont montré des masses hyperémiques solides, échogènes et bien délimitées, avec des pédicules vasculaires proéminents typiques des ganglions lymphatiques hypertrophiés (figure 1 et annexe 1 , accessible en anglais au www.cmaj.ca/lookup/doi/10.1503/cmaj.202663/tab-related -content). Les résultats des analyses sérologiques visant le virus Epstein-Barr, le cytomégalovirus, les adénovirus, les mycoplasma, la toxoplasmose, la syphilis et le VIH se sont révélés négatifs. L'analyse par séquençage métagénomique de nouvelle génération à partir d'un échantillon de sang entier a montré la présence d'une infection à Bartonella henselae. Le patient ne tolérant pas l'azithromycine orale, nous l'avons traité au moyen d'azithromycine intraveineuse ( $10 \mathrm{mg} / \mathrm{kg} / \mathrm{j}, 1$ fois par jour) pendant 7 jours. Sa fièvre s'est rapidement résorbée et ses ganglions lymphatiques ont graduellement diminué de volume. L'enfant est resté bien et n'a signalé aucun autre symptôme lors des suivis périodiques.

La maladie des griffes du chat, une zoonose présente partout dans le monde causée par les bactéries à Gram négatif du genre $B$. henselae, est caractérisée par une lymphadénopathie régionale bénigne spontanément résolutive qui s'installe d'une à 8 semaines après une griffure ou une morsure de chat domestique ${ }^{1,2}$. Environ 22000 cas de maladie des griffes du chat sont diagnostiqués chaque année aux ÉtatsUnis, surtout chez les enfants ${ }^{3,4}$. II ne s'agit pas d'une maladie

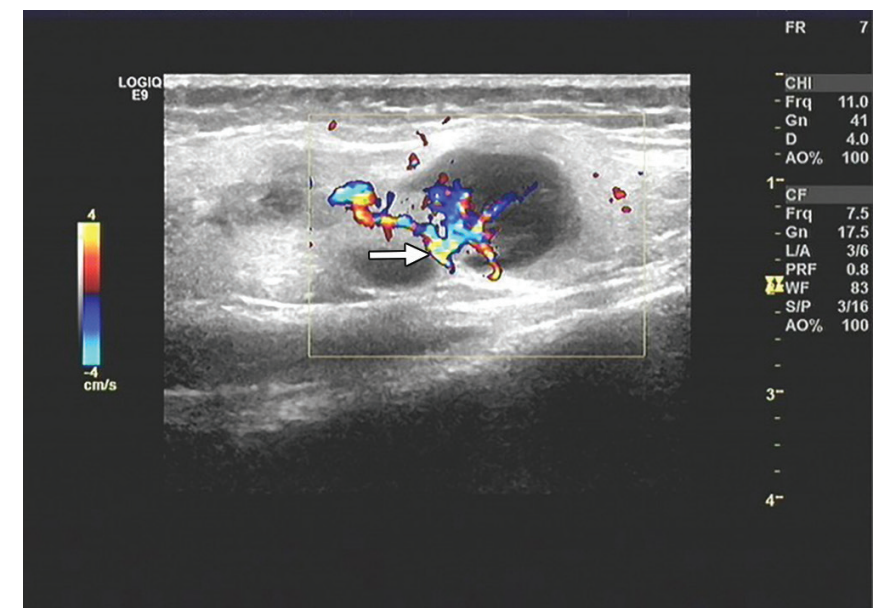

Figure 1 : Échographie Doppler de la partie supérieure du bras droit d'un garçon de 10 ans montrant des masses hyperémiques solides, échogènes et bien délimitées, avec des pédicules vasculaires proéminents (flèche) typiques des ganglions lymphatiques supratrochléaires hypertrophiés. La masse la plus volumineuse mesure $2,4 \times 1,5 \mathrm{~cm}$.

à déclaration obligatoire. Les antibiotiques ne sont pas recommandés dans les cas légers, mais de l'avis des experts, l'azithromycine est indiquée chez les cas modérés et les patients immunosupprimés ${ }^{4,5}$. Les médecins devraient envisager la possibilité de maladie des griffes du chat lors de l'établissement du diagnostic différentiel d'une lymphadénopathie régionale ou unilatérale.

\section{Références}

1. Eidlitz-Markus T, Zeharia A. Images in clinical medicine. Cat scratch disease lymphadenopathy. N Engl J Med 2006;354:e17.

2. Rohr A, Saettele MR, Patel SA, et al. Spectrum of radiological manifestations of paediatric cat-scratch disease. Pediatr Radiol 2012;42:1380-4.

3. Barson WJ, Honegger JR, Texter K. Acute myopericarditis associated with cat scratch disease in an adolescent. Pediatr Infect Dis J 2014;33:982-4.

4. Nelson CA, Moore AR, Perea AE, et al. Cat scratch disease: U.S. clinicians' experience and knowledge. Zoonoses Public Health 2018;65:67-73.

5. Rolain JM, Brouqui P, Koehler JE, et al. Recommendations for treatment of human infections caused by Bartonella species. Antimicrob Agents Chemother 2004;48:1921-33. 
Intérêts concurrents : Aucun déclaré.

Cet article a été révisé par des pairs.

Les auteurs ont obtenu le consentement des parents.

Affiliations : Département de pédiatrie (Chen, Li), Premier hôpital du Peuple de Jiashan, Jiashan, Zhejiang, Chine; Département de pneumologie (Ding), Hôpital pédiatrique de Shanghai, Université Jiao Tong de Shanghai, Shanghai, Chine.

Propriété intellectuelle du contenu : Il s'agit d'un article en libre accès distribué conformément aux modalités de la licence Creative Commons Attribution (CC BY-NC-ND 4.0), qui permet l'utilisation, la diffusion et la reproduction dans tout médium à la condition que la publication originale soit adéquatement citée, que l'utilisation se fasse à des fins non commerciales (c.-à-d., recherche ou éducation) et qu'aucune modification ni adaptation n'y soit apportée. Voir : https://creativecommons.org/licenses/by-nc-nd/4.0/deed.fr.

Remerciements : Les auteurs remercient la $D^{\text {re }}$ Angela Vinturache pour ses commentaires essentiels et sa révision substantielle de ce rapport.

Correspondance : Guodong Ding, dingguodong@shchildren.com.cn

Les images cliniques sont choisies pour leur caractère particulièrement intéressant, classique ou impressionnant. Toute soumission d'image de haute résolution claire et bien identifiée doit être accompagnée d'une légende aux fins de publication. On demande aussi une brève explication (300 mots maximum) de la portée éducative des images, et des références minimales. Le consentement écrit du patient au regard de la publication doit être obtenu avant la soumission. 